\title{
The Role of Interstitial Cells of Cajal (ICC) in Gastrointestinal Motility Disorders - What the Gastroenterologist Has to Know
}

\author{
Christian Breuer \\ Clinic of General Pediatrics, \\ Department of Pediatric Gastroenterology, \\ University Children's Hospital Hamburg-Eppendorf,
}

Germany

\section{Introduction}

Undisturbed gastrointestinal motility or peristalsis is critical for effective digestion and absorption of our food and its ingredients. Peristaltic waves in the gut are propelled by the circular and longitudinal layers of smooth muscle cells in the different segments of the gastrointestinal tract. The initiation and regulation of peristalsis is a complex process which, secondary to smooth muscle cells and enteric nerves, involves pacemaker cells of mesenchymal origin called interstitial cells of Cajal (ICC).

ICC form a network, which is widely distributed in all layers of the gastrointestinal tract. In recent years, there is growing evidence that ICC serve as electrical pacemakers and generate spontaneous electrical slow waves which constitute the basic electrical rhythm in the gastrointestinal tract. Additionally, they are important for the active propagation of slow waves and mediate neurotransmission between inhibitory and excitatory enteric neurons and smooth muscle cells.

Despite their first description more than 100 years ago, only in recent years their importance for the regulation of smooth muscle activity has been discovered. By now the presence of ICC or ICC-like cells has been demonstrated in most visceral organs which generate spontaneous rhythmic muscle contractions, e.g. the urinary and genital system.

The foremost aim of this chapter will be to inform the reader about the recent insights in the role of ICC for the physiologic course of gastrointestinal motility. A short overview about anatomical morphology, signal transduction, physiology and function will be given.

Until recently only little was known about the relevance of ICC as a pathogenetic factor for specific gastrointestinal motility diseases. By now, loss or dysfunction of ICC networks has been associated with slow transit constipation, idiopathic megacolon, diabetic gastropathy, and other diseases of impaired gastrointestinal motility. This chapter will give a short overview about the recent knowledge and highlight the role of ICC in the pathogenesis of gastrointestinal motility disorders. 


\section{History and morphology of interstitial cells of Cajal and the ICC network}

Long lasting constipation is a common problem for up to $10 \%$ of the population in the western world. Especially children and elderly people are affected. The symptom of constipation can be the result of many different anatomical, metabolic or functional disorders. For decades physicians and scientists saw the interaction between the enteric nerve system and the smooth muscle cells of the gut as the central point for regulation of sound peristalsis, facilitating proper segmentation and absorption of food and nutrients. Only during the last two decades it became obvious, that the core unit that controls gastrointestinal motility also includes ICC.

For more than 100 years ICC have been known to the histologists as mesenchymal cells ubiquitously distributed within the tunica muscularis of the bowel. The later Nobel prize laureate Santiago Ramón y Cajal first described the cells that are located interstitially between nerve endings and smooth muscle cells (Cajal, 1893). They form a network, which is found in all layers of the gastrointestinal tract. They represent approximately $5 \%$ of cells that make up the tunica muscularis and develop independently of neuronal crest-derived enteric neurons or glia from mesenchymal precursor cells. Although we know almost nothing about the metabolism, differentiation, and regeneration of these cells, a dense, comprehensive network of these spindle-shaped cells can be found in the newborn as well as in elderly people.

The distribution of ICC varies between the different layers and different segments of the gastrointestinal tract. Myenteric ICC (ICC-MY) form dense networks and are closely located to the myenteric plexus between the circular and longitudinal layers of the tunica muscularis. More loosely scattered ICC are found in the deep muscular plexus (ICC-DMP) and even in the submucosa of the stomach and colon. Intramuscular ICC within the circular and longitudinal layers of muscle in the gastrointestinal tract (ICC-IM) are closely associated with nerve varicosities throughout the muscularis propria. The tight contact of ICC with nerve cell varicosities and smooth muscle cells prompted theories they might conduct signal transmission between the gastrointestinal nerve system and smooth muscle cells. Already Ramón y Cajal himself suspected their involvement in transduction of nerve impulses after recognizing the close associations between ICC, enteric neurons, and smooth muscle cells. He used methylene blue stains when he characterized the cells at the beginning of the twentieth century so well, that they still bear his name (Cajal, 1911).

Typical ICC are defined through their spindle-shaped, elongated body with several branches and processes. The nucleus is ovoid and a basal lamina and a thick capsula made of collagen may envelope the cells (Faussone-Pellegrini and Thuneberg, 1999). With the support of modern electron microscopy more ultrastructural details of these cells have been defined and they could now be divided into at least three different types by comparing their intracellular characteristics. These include abundant mitochondria, endoplasmatic reticulum and intermediate filaments, whereas contractile proteins are missing. Frequent gap junctions are seen between the ICC to form the network through the bowel wall (Streutker et al., 2007).

In recent years the search for different specific markers for ICC has resulted in a rapidly increasing number of approaches applicable for light and fluorescence microscopy. The new interest in ICC increased rapidly twenty years ago, when Hitomi Maeda established an 
easy-to-use marker protein for ICC, identifying ICC as Kit-expressing cells using an anti-Kit antibody (Maeda et al., 1992). The protein encoded by $c$-kit is the receptor tyrosin kinase Kit

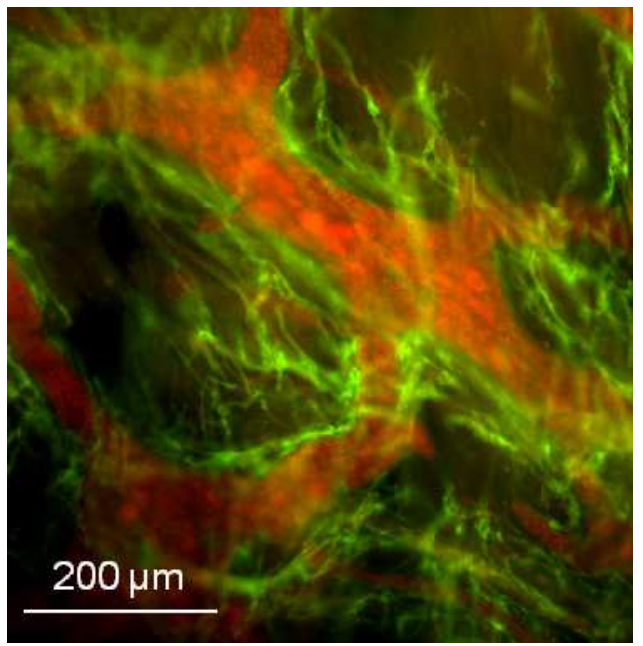

Fig. 1. Normal morphology of c-Kit stained ICC and PGP-positive myenteric neurons in human ileum. Note the dense network of ICC linked by many branches and processes overlying the myenteric ganglion (staining for the neuronal marker PGP 9.5, red; PH164, The Binding Site, Birmingham, UK; staining for c-Kit, green; PC34, Oncogene, Boston, MA, USA).

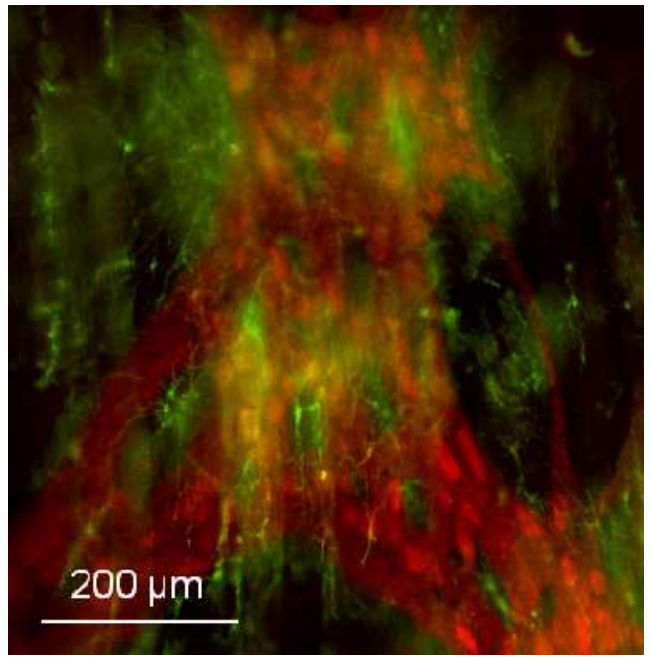

Fig. 2. Abnormal morphology of myenteric ICC and enteric neurons in the colonic biopsy of an adolescent with slow transit constipation. Note the reduced density and ramification of the ICC network. Also remarkable are the gaps in the ganglion structure, which are highly uncommon in young patients. 
(CD117) which is critical for development and function of ICC. Blockade of c-Kit function resulted in mice lacking ICC in the gastrointestinal wall. With electrophysiological examinations Maeda et al. revealed that the slow wave of the guts peristalsis was missing in the animals affected bowel segments. These findings suggested that ICC and c-Kit play a crucial role in the development of the pacemaker system that is required for the generation of autonomic gut motility. This discovery modified the concept of how gastrointestinal motility is controlled dramatically by suggesting that smooth muscle cells need more than just impulses from enteric nerve cells to generate organized gastrointestinal motility. Meanwhile, it is well established that ICC serve as electrical pacemakers and generate spontaneous electrical slow waves in the gastrointestinal tract (Takaki, 2003; Ward et al., 2004). Slow waves organize gut contractions into phasic contractions that are the basis for peristalsis and segmentation.

\section{Physiology and function of ICC}

The motility of the gastrointestinal tract drives its contents towards the aboral parts of the gut. Local pendular, non-propulsive mixing and propulsive peristaltic waves are taking part in this process. Still, the control of these movements is poorly understood. The intrinsic enteric nervous system consists of more than 100 million neurons. That is approximately the same amount than can be found in the spinal cord. The enteric nerve system therefore represents a complex integrative system which processes sensoric input and motoric output on a highly sophisticated level. As we know today, the regulation by motor neurotransmission is much more complicated than simple release of transmitter from nerve terminals and binding of receptors on smooth muscle cells.

A central part of the gastrointestinal motility regulation are ICC which are closely connected to smooth muscle cells and enteric neurons by gap junctions. They generate rhythmic pacemaker activity which triggers the slow depolarizing membrane potentials accompanying the contractions in smooth muscle cells. The term "slow waves" was coined because of their low frequency of occurrence and long duration (Mostafa et al., 2010). As recent studies confirm, ICC-MY are the specific pacemaker cells in the gut. Even after blocking the gut's neuronal function completely, recordings from isolated muscle cells show a regular pacemaker pattern generated by ICC. The pacemaker potentials generated by ICCMY are passively propagated to the smooth muscle cells via gap junctions to generate slow waves (Huang and $\mathrm{Xu}, 2010)$. Special ICC subpopulations can transfer pacemaker depolarisation from ICC-MY deep into the distant bundles of circular muscle, orchestrating contractile patterns such as the spreading ring of contraction in peristalsis or the alternating contractions of segmentation (Sanders and Ward, 2007).

Slow wave driven peristalsis varies between the segments of the gastrointestinal tract. Whereas in the stomach a primarily unidirectional peristalsis is present, the colon shows a digestive activity which is much more sensitive to modulation by distension or neuronal input. The exact mechanisms for the generation of pacemaker currents still remain controversial. The activity of a pacemaker channel is required, to initiate the pacemaker current. Many studies have shown that inhibitors of calcium channels like cyclopiazonic acid inhibited slow waves and pacemaker currents in ICC (Ward et al., 2000). Therefore, intracellular calcium seems to play an important role in the pacemaker activities. Meanwhile more and more evidence has been gathered that changes in intracellular calcium levels activate non-selective cation channels and/or calcium-activated chloride channels to 
conduct the depolarizing pacemaker currents in ICC (Takaki et al., 2010). The frequency of the generated slow wave determines the contractile frequency of the gut.

Additionally to acting as a mere pacemaker for the guts peristalsis, ICC seem to play an important role in transducing inputs from enteric motor neurons. Ultrastructural studies have found synapse-like membrane densities between ICC-IM and enteric nerve terminals. Accessorily, a variety of receptors for neurotransmitters have been identified in ICC. In murine fundus it was shown that ICC-IM were closely associated with cholinergic neurons (Huang and $\mathrm{Xu}, 2010)$. These and other results suggest that ICC-IM mediate neurotransmission and signalling between autonomic nerves and smooth muscle cells in the gut (Takaki et al., 2010). Both of the dominant enteric neural motor inputs, i.e. cholinergic and nitrergic, can influence slow wave frequency through communication with ICC (Ward et al., 2004).

The recent data confirms the complexity of gastrointestinal motility and credits ICC with at least three major regulative functions: $1^{\text {st }}$ generating of spontaneous electrical rhythms of the gut known as slow waves, $2^{\text {nd }}$ creating a propagation pathway for slow waves to adjacent parts of the guts musculature, and $3^{\text {rd }}$ mediating excitatory cholinergic and inhibitory nitrergic neuronal inputs from the enteric nervous system. Finally, some novel studies indicate that ICC may serve as nonneural stretch receptors in gut muscle, affecting both smooth muscle excitability and slow wave frequency (Quigley, 2010). Considering the immense effects of ICC on intestinal motility, it should be no surprise that ICC have been in the focus of attention in various gastrointestinal motility disorders. In fact, decreased ICC numbers and abnormal integrity are meanwhile considered a hallmark in diabetic gastropathy as well as in slow transit constipation (Farrugia, 2008). Alterations in the ICC network are thought to have a dramatic effect on the motility of the gastrointestinal system.

\section{The role of ICC in gastrointestinal motility disorders}

Many gastrointestinal motility disorders have been associated with abnormal numbers or disorders of ICC so far (Burns, 2007; Farrugia, 2008; Ordog et al., 2009). For the clinical workup it is important to keep in mind that no reference values for ICC numbers or network structure have been published. This is due to the different types of biopsy preparations and stainings in use. Therefore, study results have to be interpreted with great care, and comparisons of results from different laboratories can be misleading. For the visualisation of c-Kit (CD117), a receptor tyrosine kinase expressed by ICC, a variety of antibodies is available, yielding results of different quality. Not until 2009 an optimized protocol for improved ICC detection in gastrointestinal motility disorders was presented (Garrity et al., 2009). Since ICC appear very sensitive to hypoxia, abnormalities noted on pathological examination of specimen may also reflect tissue ischemia prior to fixation (Farrugia, 2008).

Most of the ideas regarding the cause of several gastrointestinal motor disorders are still derived from animal models. Because human biopsy samples are still rarely obtained for ICC staining, most case reports published in the literature are describing only a few cases each. However, meanwhile several papers, using a variety of techniques, describing abnormal ICC networks in gastrointestinal motility disorders, have been published. A pubmed search with the keywords "interstitial cells of Cajal" and "gastrointestinal motility" now yields more than 400 results. Whether the observed changes in ICC morphology are primary, secondary or merely epiphenomic remains still much debated (Gladman and 
Knowles, 2008). Some authors propose that changes in ICC networks in gastrointestinal diseases are due to inflammatory processes or mechanically induced due to long lasting intestinal obstruction. In a number of human motility disorders with abnormal ICC, however, there is no overt inflammation or dilatation suggesting that this cannot be the only reason for the observed ICC abnormalities (Farrugia, 2008).

\subsection{Achalasia}

ICC of the intramuscular subtype are found in the muscular layers of the oesophagus and especially in the lower oesophageal sphincter (LES). Ultrastructural damage and loss of ICCIM in the oesophagus have been reported in achalasia (Faussone-Pellegrini and Cortesini, 1985; Streutker et al., 2007). The disease is characterized by an impaired relaxation of the LES, leading to dilatation of the proximal segments of the oesophagus. Etiologically a loss of inhibitory nerves and progressive degeneration of ganglion cells containing vasoactive intestinal peptide (VIP) and nitric oxide (NO) are suspected to be major causes of LES dysfunction (Negreanu et al., 2008). The pathogenetic role of ICC in achalasia is still much debated. Sanders et al. suggest that loss of ICC in the LES would lead to loss of cholingergic tone and changes in LES tension subsequently (Sanders et al., 2002). Although the close anatomical relationship between ICC and cholinergic neurons is well established in electron microscopy, appropriate studies to determine the functional effect of ICC on the regulation of LES relaxation are still lacking.

\subsection{Gastroesophageal reflux disease (GERD)}

GERD is one of the most common causes for patients to visit a gastroenterologist. The major mechanism is a transient relaxation of the LES (Kaltenbach et al., 2006). Typical symptoms of heartburn and regurgitation are temporarily encountered by almost $20 \%$ of the general adult population. However, no specific histopathological abnormalities of ICC have been found and described so far in humans. Animal studies with ICC lacking $\mathrm{W} / \mathrm{W}^{\mathrm{v}}$ mice showed a normal swallow induced LES relaxation, contradicting a significant role of ICC in the pathogenesis of reflux disease (Dickens et al., 2001). Secondary changes to the ICC architecture may be induced, when GERD is complicated by severe oesophagitis, oesophageal ulcers or Barret's oesophagus (Negreanu et al., 2008).

\subsection{Diabetic gastroenteropathy}

Gastroparesis is defined as slow gastric emptying which could not be explained by mechanical obstruction. Up to $30-60 \%$ of diabetic patients develop associated symptoms, e.g. dysphagia, heartburn, nausea, abdominal pain, or discomfort. In the long term, gastroparesis may lead to more severe symptoms like nutritional insufficiency, electrolyte imbalance, and impaired glycaemic control (Koch, 1999). The pathomechanisms leading to diabetic gastroparesis are complex and are usually interpreted as a consequence of damage to the autonomic nerve system. Only recently studies addressed the role of ICC loss and dysfunction in the pathophysiology of diabetic gastroenteropathy (Ordog, 2008).

Well characterized animal models have been established for the research of diabetes mellitus type 1 . In studies with non-obese diabetic mice (NOD-mice), Ordog et al. showed clearly reduced ICC networks by c-Kit immunofluorescence and electron microscopy in the 
gastric corpus and antrum in comparison with controls. Both ICC-IM and ICC-MY were affected. Additionally, myenteric ICC were also reduced in the colon. In humans ICC loss in the stomach has also been well documented. In four of nine patients with diabetic gastroparesis profound loss of ICC was observed in biopsy samples of the gastric wall. In another study the same authors reported that nine of 23 patients with gastroparesis showed complete absence of ICC in their antrum biopsies (Forster et al., 2005).

Whether ICC of the small intestine and colon are also affected in humans with diabetes is controversial. Human data is still sparse and patients often suffer from additional maladies such as colonic cancer, which may lead to misinterpretations of the data. In rodent models, however, changes in ICC morphology could also be observed in the proximal and distal colon. However, slow wave frequencies, velocities and extracellular amplitudes were unchanged in a recent study with streptozotocin treated diabetic rats (Lammers et al., 2011), challenging the hypothesis that ICC reduction is severe enough to effect slow wave propagation in the bowel.

Regarding the stomach, the question is, whether the demonstrated changes in ICC morphology are somehow related to the impairment of gastric function in diabetic gastropathy. Delayed emptying of nutrient liquids and solids is caused primarily by antral hypomotility. Reduced smooth muscle contractions as a result of diabetic myopathy and a reduction of pacing electrical slow waves may contribute to the gastric stasis. The role of ICC in the generation and propagation of gastric electrical slow waves is well-established (Ordog, 2008). Abnormal slow wave activity has been shown in different rodent models for diabetes type 1 and 2 combined with diffuse reduction of ICC (Takano et al., 1998). Diabetic rodents showed signs of gastroparesis as well as gastric dysrhythmias. The electrical abnormalities resemble those typically found in human diabetic patients. Thus, reduction in ICC most likely plays a key role in the pathogenesis of diabetic gastroparesis by contributing to gastric hypomotility and delayed gastric emptying.

\subsection{Infantile hypertrophic pyloric stenosis}

Pyloric stenosis is a congenital disorder characterized by hypertrophy of the inner muscle ring of the pylorus. Signs of delayed gastric emptying are usually not present at birth. Vomiting often starts around the age of three weeks. The etiology is unknown, but abnormal innervation of the pyloric muscle has been implicated. Some studies showed absence of ICC in the hypertrophic circular muscle layer in infants with hypertrophic pyloric stenosis, along with loss of peptidergic and nitric oxide containing nerves (Vanderwinden and Rumessen, 1999). The authors propose the loss of ICC may contribute to the lack of antropyloric coordination and therefore aggravate vomiting and the proper passage of food through the pylorus. An interesting but still unproven theory is that delayed ICC maturation might be one reason explaining the lack of ICC found in immunohistochemnistry and electron microscopy. However, more data is needed to specify the role of ICC in the pathogenesis of hypertrophic pyloric stenosis.

\subsection{Slow transit constipation (STC)}

Patients suffering from STC show a normal gut diameter and have a prolonged colonic transit. In the majority of cases STC is of unknown etiology. However, it is now well established that ICC play an important role in the pathophysiology of STC. It is plausible 
that a loss of pacemaker cells would be associated with decrease in colonic transit. Studies proved that ICC density in the colon of patients was significantly decreased compared with those of normal patients (He et al., 2000; Lyford et al., 2002). Expression of $c-k i t$ mRNA and c-Kit protein in the colon were also decreased in STC, suggesting an important role of the cKit signal pathway in the ICC reduction (Tong et al., 2005). Remarkably, these changes in ICC morphology are often accompanied with loss of enteric neurons (Lee et al., 2005) emphasizing the very close relationship between ICC and intrinsic nerves and glial cells. However, a recent study using an automated cellular imaging system for immunohistochemical detection of c-Kit (CD117) showed no significant depletion of ICC. The authors propose that the mere quantification of ICC numbers in the bowel wall may not provide sufficient information on the functional status, since mediation from cholinergic and nitrergic nerves must also be intact for correct ICC function (Toman et al., 2006). Nevertheless, the observed changes in ICC numbers and integrity in patients with STC suggest an important role of ICC when elucidating reduced gastrointestianal motor activity. This has already led some pathology laboratories to include a stain for c-Kit as part of their routine evaluation of specimens resected from patients with STC (Garrity et al., 2009). Future studies will help to understand the exact physiologic and pathopysiologic nature of ICC in STC.

\subsection{Chronic intestinal pseudo-obstruction}

The term chronic intestinal pseudo-obstruction is used for a group of disorders showing symptoms of intestinal obstruction in the absence of any anatomical or mechanical lesion. Underlying the disease are pathologic disorders from intestinal neuropathy to intestinal myopathy or both. Absence of ICC has been already suggested to be a causative factor leading to intestinal pseudo-obstruction in adults (Vanderwinden and Rumessen, 1999). Yamataka et al. reported abnormal distribution of myenteric ICC in an infant using immunohistochemistry with antihuman c-Kit serum (Yamataka et al., 1998). Recently, one child with total absence of ICC in the myenteric plexus of the distal ileum and colon was reported (Struijs et al., 2008). Feldstein showed altered networks of ICC in a 14-year-old boy (Feldstein et al., 2003). Additionally, delayed maturation of ICC, with normalisation of ICC numbers in subsequent biopsy specimen, has been reported in cases of neonatal pseudoobstruction (Kenny et al., 1998b).

These findings strongly suggest an important etiologic role for ICC at least in some patients with intestinal pseudo-obstruction. Although many more factors may contribute to manifestation and presentation of the disease, the existent data demonstrates that abnormalities of ICC should be considered early in the diagnostic workup of children with intestinal pseudo-obstruction.

\subsection{Congenital aganglionic megacolon (Hirschsprung's disease)}

In Hirschsprung's disease the intrinsic enteric nervous system is absent in a segment of the gastrointestinal tract. Mostly found in the distal colon, the aganglionosis may also involve large portions of the entire bowel. Some specimen from the aganglionic region of the colon showed reduced ICC numbers and damaged ICC networks (Wang et al., 2009). Other studies however, point out a high heterogeneity in ICC values within a group of Hirschsprung's patients (Bettolli et al., 2008). Hypothetically, the depletion of ICC in the 
muscular layer of the gut may contribute to the inability of the smooth muscle to relax. Together with the lack of neurons, a defective initiation of pacemaker currents may also contribute to the motility dysfunction in affected bowel segments. Nevertheless, Hirschsprung's disease remains a heterogenous and multigenetic disease and a routine immunohistochemnistry for ICC seems not helpful in differentiation between the healthy and the aganglionic part of the bowel today. Genetic evidence recently confirmed already the absence of linkage between hereditary forms of Hirschsprung's disease and the region of the ICC-regulating gene $c-k i t$ (Dow et al., 1994; Mostafa et al., 2010).

\subsection{Idiopathic megacolon}

In contrast to the congenital megacolon known as Hirschsprung's disease, patients with acquired megacolon show no aganglionic bowel segments and the enteric innervation seems to be intact. Thus, it had been proposed that colonic dysmotility in these patients might as well result from alterations of ICC. Accordingly, some studies of patients with idiopathic megabowel showed decreased ICC density (Lee et al., 2005; Wedel et al., 2002). By contrast, another study of sixty-three patients with megacolon showed no consistent alterations in colonic ICC histology (Meier-Ruge et al., 2006). The results suggest once again that ICC might play an important etiologic role, however more systematic studies are needed to determine the detailed pathomechanisms.

\subsection{Children with anorectal malformations}

Anorectal malformations comprise a wide spectrum of diseases, which involve the distal anus and rectum as well as the urinary and genital tracts. Defects range from minor anal anomalies to very complex cloacal malformations, which are often associated with other anomalies (Levitt and Pena, 2007). The etiology of such malformations remains unclear and is likely multifactorial. However, in recent years a dramatic progress in operation techniques has improved prognosis significantly.

Constipation, eventually leading to megarectum and functional outlet obstruction, is a common postsurgical problem faced by more than a half of all patients with anorectal malformations. Although in some of these patients aganglionosis or neuronal intestinal dysplasia have been reported, no plausible theory could explain colonic hypomotility satisfactorily so far. Often these symptoms have been attributed to associated abnormalities of the sacral roots or to inherent abnormalities of the myenteric plexus.

Using monoclonal mouse antibody against c-Kit, Kenny et al. reported marked abnormalities in density and distribution of c-Kit-positive ICC in the sigmoid colon in 7 of 12 patients with high or intermediate anorectal malformations (Kenny et al., 1998a). Since no electron microscopy was applied, the authors state that it is uncertain, if the loss of c-Kit immunoreactivity was caused by phenotypic loss of c-Kit antigen, or by complete absence of ICC. The latter would advance speculations that genes involved in gut segmentation and hindgut differentiation are also essential for ICC development. Supporting this, a lower density of ICC in the terminal intestine was recently observed in rats with ethylenethioureainduced anorectal malformations (Macedo et al., 2008). Although not enough sufficient data is available today, congenital defects in interstitial pacemaker cells may, additionally to other factors, contribute to the colonic hypomobility in patients with anorectal malformations. 


\subsection{Patients with mutations of c-kit and constipation}

Signals through the c-Kit receptor tyrosine kinase are essential for development and differentiation of erythrocytes, melanocytes, germ cells, mast cells, and ICC. Gain-offunction mutations of $c$-kit result in the development of mast cell and germ cell tumors, and of ICC tumors called gastrointestinal stromal tumors (GIST), respectively (Kitamura and Hirotab, 2004). In mice the $\mathrm{W}$ locus was demonstrated to encode c-Kit, and meanwhile various types of mutants have been reported. Animal models with loss-of-function mutations in the c-kit gene compromise the regular development of ICC in the gut (Alberti et al., 2007; Maeda et al., 1992; Sanders and Ward, 2007). In humans however, no homozygote loss-of-function mutations have been reported so far.

Recently one juvenile patient was described whose biopsy specimen showed only few ICC in the ileum and complete absence of ICC in the colon (Breuer et al., 2010). The mutational analysis of Kit in this patient revealed multiple genetic alterations at the level of mRNA which potentially could result in a loss of function of the Kit protein. According to this, it was suggested that the genetic alterations of $c-k i t$ might lead to alterations in ICC architecture and function.

By now animal studies have already shown that point mutations in the proto-oncogene $c$-kit correlate with abnormal intestinal contractions in vitro (Isozaki et al., 1995). Kit mutations might therefore explain cases of patients with megacolon or small transit constipation who show histopathological defects or depletion of ICC. The Wsh $/ W^{\text {sh }} \mathrm{c}-\mathrm{Kit}$ mutant illustrates the complexity of Kit-regulated ICC differentiation. Although leading to a general absence of ICC in the intestine due to an inversion mutation upstream the promoter region, a subpopulation of special ICC in the deep muscular plexuses (ICC-DMP) developed normally in mutant mice. These findings suggest that ICC-DMP may develop and differentiate without c-Kit expression (Iino et al., 2009).

\section{Summary}

Constipation and fecal impaction are frequent and distressing complaints in gastroenterology. In most cases a sufficient treatment including changes in lifestyle, activity, and food, with the additional use of laxatives is possible. However, the treatment of severe forms of constipation may constitute a difficult task.

In recent years the role of ICC in gastrointestinal motility is increasingly recognized. Throughout the whole gastrointestinal tract ICC-MY and ICC-IM coordinate smooth muscle activity and guarantee the physiologic course of peristalsis. Whereas ICC-MY act as pacemaker cells to generate slow waves driven by changes of voltage dependent calcium channels, ICC-IM mediate neurotransmission between enteric neurons and smooth muscle cells. Meanwhile lots of cases with histological alterations in ICC morphology have been presented in patients with different types of constipation. However, it occasionally remains unclear, whether morphological alterations of ICC are based on congenital developmental anomalies or whether they are a consequence of long term constipation with secondary damage of the guts neuroarchitecture. Nevertheless, the new insights in ICC physiology and function present a new aspect for gastroenterologists to focus on, when dealing with patients suffering from severe forms of constipation. Clinicians should consider involvement of ICC early in the diagnostic process of motility disorders. Further 
investigations may lead to the routine staining and evaluation of ICC-morphology in intestinal biopsy specimen.

Although the knowledge of the role of ICC in gastrointestinal disorders is increasing rapidly, no major progress has been achieved in treatment so far. The Kit inhibitor Imatinib mesylate has been shown to be effective in Kit expressing tumors (GISTs), but no drugs improving loss of c-Kit function are available today. Eventually new medications modulating gastrointestinal peristalsis may be provided in the future. Replacement of defective pacemaker cells however, will be a prospective promise of genetic therapy at best.

\section{References}

Alberti, E., Mikkelsen, H. B., Wang, X. Y., Diaz, M., Larsen, J. O., Huizinga, J. D., and Jimenez, M. (2007). Pacemaker activity and inhibitory neurotransmission in the colon of Ws/Ws mutant rats. Am J Physiol Gastrointest Liver Physiol 292, G14991510.

Bettolli, M., De Carli, C., Jolin-Dahel, K., Bailey, K., Khan, H. F., Sweeney, B., Krantis, A., Staines, W. A., and Rubin, S. (2008). Colonic dysmotility in postsurgical patients with Hirschsprung's disease. Potential significance of abnormalities in the interstitial cells of Cajal and the enteric nervous system. J Pediatr Surg 43, 14331438.

Breuer, C., Oh, J., Molderings, G. J., Schemann, M., Kuch, B., Mayatepek, E., and Adam, R. (2010). Therapy-refractory gastrointestinal motility disorder in a child with c-kit mutations. World J Gastroenterol 16, 4363-4366.

Burns, A. J. (2007). Disorders of interstitial cells of Cajal. J Pediatr Gastroenterol Nutr 45 Suppl 2, S103-106.

Cajal, S. R. (1893). Sur les ganglions et plexus nerveux d'intestin. C R Roc Biol Paris 5, 217223.

Cajal, S. R. (1911). Histology of the nervous system of man and vertebrates. translation by Swanson and Swanson, Oxford University Press 1995, 891-942.

Dickens, E. J., Edwards, F. R., and Hirst, G. D. (2001). Selective knockout of intramuscular interstitial cells reveals their role in the generation of slow waves in mouse stomach. J Physiol 531, 827-833.

Dow, E., Cross, S., Wolgemuth, D. J., Lyonnet, S., Mulligan, L. M., Mascari, M., Ladda, R., and Williamson, R. (1994). Second locus for Hirschsprung disease/Waardenburg syndrome in a large Mennonite kindred. Am J Med Genet 53, 75-80.

Farrugia, G. (2008). Interstitial cells of Cajal in health and disease. Neurogastroenterol Motil 20 Suppl 1, 54-63.

Faussone-Pellegrini, M. S., and Cortesini, C. (1985). The muscle coat of the lower esophageal sphincter in patients with achalasia and hypertensive sphincter. An electron microscopic study. J Submicrosc Cytol 17, 673-685.

Faussone-Pellegrini, M. S., and Thuneberg, L. (1999). Guide to the identification of interstitial cells of Cajal. Microsc Res Tech 47, 248-266.

Feldstein, A. E., Miller, S. M., El-Youssef, M., Rodeberg, D., Lindor, N. M., Burgart, L. J., Szurszewski, J. H., and Farrugia, G. (2003). Chronic intestinal pseudoobstruction associated with altered interstitial cells of cajal networks. J Pediatr Gastroenterol Nutr 36, 492-497. 
Forster, J., Damjanov, I., Lin, Z., Sarosiek, I., Wetzel, P., and McCallum, R. W. (2005). Absence of the interstitial cells of Cajal in patients with gastroparesis and correlation with clinical findings. J Gastrointest Surg 9, 102-108.

Garrity, M. M., Gibbons, S. J., Smyrk, T. C., Vanderwinden, J. M., Gomez-Pinilla, P. J., Nehra, A., Borg, M., and Farrugia, G. (2009). Diagnostic challenges of motility disorders: optimal detection of CD117+ interstitial cells of Cajal. Histopathology 54, 286-294.

Gladman, M. A., and Knowles, C. H. (2008). Novel concepts in the diagnosis, pathophysiology and management of idiopathic megabowel. Colorectal Dis 10, 531-538; discussion 538-540.

He, C. L., Burgart, L., Wang, L., Pemberton, J., Young-Fadok, T., Szurszewski, J., and Farrugia, G. (2000). Decreased interstitial cell of cajal volume in patients with slowtransit constipation. Gastroenterology 118, 14-21.

Huang, X., and Xu, W. X. (2010). The pacemaker functions of visceral interstitial cells of Cajal. Sheng Li Xue Bao 62, 387-397.

Iino, S., Horiguchi, K., and Nojyo, Y. (2009). W(sh)/W(sh) c-Kit mutant mice possess interstitial cells of Cajal in the deep muscular plexus layer of the small intestine. Neurosci Lett 459, 123-126.

Isozaki, K., Hirota, S., Nakama, A., Miyagawa, J., Shinomura, Y., Xu, Z., Nomura, S., and Kitamura, Y. (1995). Disturbed intestinal movement, bile reflux to the stomach, and deficiency of c-kit-expressing cells in Ws/Ws mutant rats. Gastroenterology 109, 456-464.

Kaltenbach, T., Crockett, S., and Gerson, L. B. (2006). Are lifestyle measures effective in patients with gastroesophageal reflux disease? An evidence-based approach. Arch Intern Med 166, 965-971.

Kenny, S. E., Connell, M. G., Rintala, R. J., Vaillant, C., Edgar, D. H., and Lloyd, D. A. (1998a). Abnormal colonic interstitial cells of Cajal in children with anorectal malformations. J Pediatr Surg 33, 130-132.

Kenny, S. E., Vanderwinden, J. M., Rintala, R. J., Connell, M. G., Lloyd, D. A., Vanderhaegen, J. J., and De Laet, M. H. (1998b). Delayed maturation of the interstitial cells of Cajal: a new diagnosis for transient neonatal pseudoobstruction. Report of two cases. J Pediatr Surg 33, 94-98.

Kitamura, Y., and Hirotab, S. (2004). Kit as a human oncogenic tyrosine kinase. Cell Mol Life Sci 61, 2924-2931.

Koch, K. L. (1999). Diabetic gastropathy: gastric neuromuscular dysfunction in diabetes mellitus: a review of symptoms, pathophysiology, and treatment. Dig Dis Sci 44, 1061-1075.

Lammers, W. J., Al-Bloushi, H. M., Al-Eisae, S. A., Al-Dhaheri, F. A., Stephen, B. S., John, R., Dhanasekaran, S., and Karam, S. M. (2011). Slow wave propagation and ICC plasticity in the small intestine of diabetic rats. Exp Physiol.

Lee, J. I., Park, H., Kamm, M. A., and Talbot, I. C. (2005). Decreased density of interstitial cells of Cajal and neuronal cells in patients with slow-transit constipation and acquired megacolon. J Gastroenterol Hepatol 20, 1292-1298.

Levitt, M. A., and Pena, A. (2007). Anorectal malformations. Orphanet J Rare Dis 2, 33.

Lyford, G. L., He, C. L., Soffer, E., Hull, T. L., Strong, S. A., Senagore, A. J., Burgart, L. J., Young-Fadok, T., Szurszewski, J. H., and Farrugia, G. (2002). Pan-colonic decrease 
in interstitial cells of Cajal in patients with slow transit constipation. Gut 51, 496501.

Macedo, M., Martins, J. L., Meyer, K. F., and Soares, I. C. (2008). Study of density of interstitial cells of cajal in the terminal intestine of rats with anorectal malformation. Eur J Pediatr Surg 18, 75-79.

Maeda, H., Yamagata, A., Nishikawa, S., Yoshinaga, K., Kobayashi, S., Nishi, K., and Nishikawa, S. (1992). Requirement of c-kit for development of intestinal pacemaker system. Development 116, 369-375.

Meier-Ruge, W. A., Muller-Lobeck, H., Stoss, F., and Bruder, E. (2006). The pathogenesis of idiopathic megacolon. Eur J Gastroenterol Hepatol 18, 1209-1215.

Mostafa, R. M., Moustafa, Y. M., and Hamdy, H. (2010). Interstitial cells of Cajal, the Maestro in health and disease. World J Gastroenterol 16, 3239-3248.

Negreanu, L. M., Assor, P., Mateescu, B., and Cirstoiu, C. (2008). Interstitial cells of Cajal in the gut--a gastroenterologist's point of view. World J Gastroenterol 14, 6285-6288.

Ordog, T. (2008). Interstitial cells of Cajal in diabetic gastroenteropathy. Neurogastroenterol Motil 20, 8-18.

Ordog, T., Hayashi, Y., and Gibbons, S. J. (2009). Cellular pathogenesis of diabetic gastroenteropathy. Minerva Gastroenterol Dietol 55, 315-343.

Quigley, E. M. (2010). What we have learned about colonic motility: normal and disturbed. Curr Opin Gastroenterol 26, 53-60.

Sanders, K. M., and Ward, S. M. (2007). Kit mutants and gastrointestinal physiology. J Physiol 578, 33-42.

Sanders, K. M., Ward, S. M., and Daniel, E. E. (2002). ICC in neurotransmission: hard to swallow a lack of involvement. Gastroenterology 122, 1185-1186; author reply 11861187.

Streutker, C. J., Huizinga, J. D., Driman, D. K., and Riddell, R. H. (2007). Interstitial cells of Cajal in health and disease. Part I: normal ICC structure and function with associated motility disorders. Histopathology 50, 176-189.

Struijs, M. C., Diamond, I. R., Pencharz, P. B., Chang, K. T., Viero, S., Langer, J. C., and Wales, P. W. (2008). Absence of the interstitial cells of Cajal in a child with chronic pseudoobstruction. J Pediatr Surg 43, e25-29.

Takaki, M. (2003). Gut pacemaker cells: the interstitial cells of Cajal (ICC). J Smooth Muscle Res 39, 137-161.

Takaki, M., Suzuki, H., and Nakayama, S. (2010). Recent advances in studies of spontaneous activity in smooth muscle: ubiquitous pacemaker cells. Prog Biophys Mol Biol 102, 129-135.

Takano, H., Imaeda, K., Koshita, M., Xue, L., Nakamura, H., Kawase, Y., Hori, S., Ishigami, T., Kurono, Y., and Suzuki, H. (1998). Alteration of the properties of gastric smooth muscle in the genetically hyperglycemic OLETF rat. J Auton Nerv Syst 70, 180-188.

Toman, J., Turina, M., Ray, M., Petras, R. E., Stromberg, A. J., and Galandiuk, S. (2006). Slow transit colon constipation is not related to the number of interstitial cells of Cajal. Int J Colorectal Dis 21, 527-532.

Tong, W. D., Liu, B. H., Zhang, L. Y., Xiong, R. P., Liu, P., and Zhang, S. B. (2005). Expression of c-kit messenger ribonucleic acid and c-kit protein in sigmoid colon of patients with slow transit constipation. Int J Colorectal Dis 20,363-367. 
Vanderwinden, J. M., and Rumessen, J. J. (1999). Interstitial cells of Cajal in human gut and gastrointestinal disease. Microsc Res Tech 47, 344-360.

Wang, H., Zhang, Y., Liu, W., Wu, R., Chen, X., Gu, L., Wei, B., and Gao, Y. (2009). Interstitial cells of Cajal reduce in number in recto-sigmoid Hirschsprung's disease and total colonic aganglionosis. Neurosci Lett 451, 208-211.

Ward, S. M., Ordog, T., Koh, S. D., Baker, S. A., Jun, J. Y., Amberg, G., Monaghan, K., and Sanders, K. M. (2000). Pacemaking in interstitial cells of Cajal depends upon calcium handling by endoplasmic reticulum and mitochondria. J Physiol 525 Pt 2, 355-361.

Ward, S. M., Sanders, K. M., and Hirst, G. D. (2004). Role of interstitial cells of Cajal in neural control of gastrointestinal smooth muscles. Neurogastroenterol Motil 16 Suppl 1, 112-117.

Wedel, T., Spiegler, J., Soellner, S., Roblick, U. J., Schiedeck, T. H., Bruch, H. P., and Krammer, H. J. (2002). Enteric nerves and interstitial cells of Cajal are altered in patients with slow-transit constipation and megacolon. Gastroenterology 123, 14591467.

Yamataka, A., Ohshiro, K., Kobayashi, H., Lane, G. J., Yamataka, T., Fujiwara, T., Sunagawa, M., and Miyano, T. (1998). Abnormal distribution of intestinal pacemaker (C-KITpositive) cells in an infant with chronic idiopathic intestinal pseudoobstruction. J Pediatr Surg 33, 859-862. 


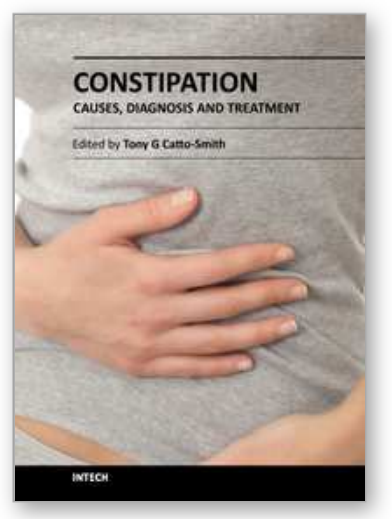

\author{
Constipation - Causes, Diagnosis and Treatment \\ Edited by Dr. Anthony Catto-Smith
}

ISBN 978-953-51-0237-3

Hard cover, 172 pages

Publisher InTech

Published online 07, March, 2012

Published in print edition March, 2012

Constipation is common in both adults and children. Estimates would suggest a median prevalence of around $12-16 \%$ in the general population. While regarded as a minor nuisance in some cases, its consequences can be severe, with a substantial impact on quality of life. Secondary faecal soiling has a profound psychological effect at all ages. This book provides contributions from authors with a range of backgrounds which clarify the pathogenesis, diagnosis, and therapy of constipation for the general population and also for certain high risk groups.

\title{
How to reference
}

In order to correctly reference this scholarly work, feel free to copy and paste the following:

Christian Breuer (2012). The Role of Interstitial Cells of Cajal (ICC) in Gastrointestinal Motility Disorders - What the Gastroenterologist Has to Know, Constipation - Causes, Diagnosis and Treatment, Dr. Anthony CattoSmith (Ed.), ISBN: 978-953-51-0237-3, InTech, Available from: http://www.intechopen.com/books/constipationcauses-diagnosis-and-treatment/the-role-of-interstitial-cells-of-cajal-icc-in-gastrointestinal-motility-disorderswhat-the-gastroen

\section{INTECH}

open science | open minds

\section{InTech Europe}

University Campus STeP Ri Slavka Krautzeka 83/A 51000 Rijeka, Croatia Phone: +385 (51) 770447 Fax: +385 (51) 686166 www.intechopen.com

\section{InTech China}

Unit 405, Office Block, Hotel Equatorial Shanghai No.65, Yan An Road (West), Shanghai, 200040, China 中国上海市延安西路65号上海国际贵都大饭店办公楼405单元 Phone: +86-21-62489820

Fax: +86-21-62489821 
(C) 2012 The Author(s). Licensee IntechOpen. This is an open access article distributed under the terms of the Creative Commons Attribution 3.0 License, which permits unrestricted use, distribution, and reproduction in any medium, provided the original work is properly cited. 